\title{
The gender-specific impact of emotional tears
}

\author{
Marie Stadel $^{1}$. Judith K. Daniels ${ }^{1,2} \cdot$ Matthijs J. Warrens $^{3} \cdot$ Bertus F. Jeronimus $^{1,4}$
}

Published online: 18 May 2019

(c) The Author(s) 2019

\begin{abstract}
The purpose of crying has recently become a topic of interest, with evidence supporting its interpersonal functions. The assumption that tears not only express a need for help, but in reaction also foster willingness to help in an observer, has received preliminary empirical support. The current study replicated previous work using a within-subject design with 140 subjects (50\% female) who were exposed to images depicting male and female individuals crying, with half of both displaying visible tears and the others not displaying tears. Novel is our comparison of willingness to help across all possible gender combinations of tear display and observer. Potential mediation by perceived helplessness, friendliness, and connectedness of the depicted person was tested in male and female participants separately. We replicated the strong effect of tears on willingness to help, and showed this effect to be less potent for male dyads than for female or mixed ones, which is new to the literature. Perceived helplessness mediated the link between crying and helping, whereas perceived connectedness seemed only relevant for female participants, and perceived friendliness was not significant. Possible origins and implications of a differential gender function of crying are discussed.
\end{abstract}

Keywords Crying $\cdot$ Helplessness $\cdot$ Connectedness $\cdot$ Friendliness $\cdot$ Gender differences

\section{Introduction}

Crying is one of the most intense emotional expressions in humans (Wassiliwizky et al. 2017). While basal tears lubricate the eyes of many animals, most psychologists ascribe the display of emotional crying or weeping as unique to humankind (Barr et al. 2014; Gračanin et al. 2018), with which many ethologists disagree (De Waal 2019; Masson

Electronic supplementary material The online version of this article (https://doi.org/10.1007/s11031-019-09771-z) contains supplementary material, which is available to authorized users.

Judith K. Daniels

j.k.daniels@rug.nl

1 Department of Clinical Psychology, University of Groningen, Grote Kruisstraat 2/1, 9712 TS Groningen, The Netherlands

2 Berlin Psychological University, Berlin, Germany

3 Groningen Institute for Educational Research, University of Groningen, Groningen, The Netherlands

4 Department of Psychiatry, Interdisciplinary Center Psychopathology and Emotion Regulation (ICPE), University of Groningen, University Medical Center Groningen, Groningen, The Netherlands and McCarthy 1995). Early evolutionary theory did not assign a function to this shedding of tears as Darwin (1872) regarded it as a purposeless by-product of evolution.

Recently, several theories on the utility of crying have been proposed and investigated (Bylsma et al. 2011; Vingerhoets et al. 2016) and the distinction between intrapersonal and interpersonal functions of crying has emerged (Denckla et al. 2014; Simons et al. 2013). Potential intrapersonal functions that have been suggested include catharsis or feelings of relief and mood improvement after tears have been shed (Rottenberg et al. 2008; Simons et al. 2013). Despite strong lay beliefs in catharsis the empirical support is insufficient (Bylsma et al. 2011). Additionally, it has been suggested that intra-personal motives for crying could relate to self-concepts, being a person who is adequately emotional (Simons et al. 2013).

Conversely, several theories on the interpersonal functions of crying have been supported by evidence (Gračanin et al. 2018): Interpersonal mechanisms that involve crying include the expression of sadness (Provine et al. 2009), a need for support, and subsequent promotion of prosocial helping behaviour (Balsters et al. 2013; Hendriks and Vingerhoets 2006). The core function of displayed tears may thus be the communication of helplessness in both positive and 
negative contexts (Cornelius and Lubliner 2003; Denckla et al. 2014; Mizokawa 2011) or to elicit appraisals of warmth (Simons et al. 2013). Similarly, crying in a social context can be seen as a form of problem- and emotion-focused coping by eliciting social support (Vingerhoets and Scheirs 2000). However, some data indicate that the display of tears might lead to various interpretations and attributions, depending on the perceiver's information about the societal context (Fischer et al. 2013). The display of tears thus needs to be considered in its context and may be best placed within the broader behavioural ecology view of facial displays (Crivelli and Fridlund 2018) and the emotion literature, which assumes that emotional expressions are best understood as social tools that are used flexibly in social interaction and evolved from animal signalling to communicate feelings (Barrett 2018; Darwin 1872).

\section{The helping hypothesis}

Vingerhoets et al. (2016) found robust initial support for this tears-elicit-help-hypothesis $(d=1.32)$ by exposing participants (Study 1: females; Study 2: males and females) to photographs of tearfully crying individuals and photos with digitally removed tears ${ }^{1}$. By manipulating tear-display they aimed to isolate the effect of the presence of tears from accompanying facial expressions. Subsequently participants were asked to indicate their willingness to help the depicted individuals (Study 1: males and females depicted; Study 2: only females depicted). Additionally, Vingerhoets et al. identified three mediators of the link between the display of tears and subsequent willingness to help: feeling connected to the displayed person (Bressan et al. 2009), and perceiving the person as helpless (Nelson 2005) and/or friendly (Hasson 2009; Hendriks et al. 2008).

Vingerhoets et al. also showed significant differences between male and female images: females were rated higher on all four measured constructs. Moreover, the effects of interactions between the display of tears and the gender of the crying person on the different constructs were explored. Hereby, no notable gender differences were observed next to a noticeable higher perceived helplessness in crying women than men. Vingerhoets et al., however, did not take all potential gender effects into account. More specifically, they did not consider the effects of observers' gender and potential interactions in cross-gender dyads. Moreover, due to their study design, it was not possible for Vingerhoets et al. to examine how male participants might react to pictures of male faces. Therefore, it remains unclear whether

\footnotetext{
1 http://www.flickr.com/photos/themuseumofmodernart/sets/72157 623741486824.
}

the tears-elicit-help effect generalizes across all gender combinations, or whether inter-gender differences are present. The current study expands the available literature with tests of the tears-elicit-help effect across all gender combinations.

\section{Gender differences in crying}

Women are known to be more likely to cry and to cry more frequently across cultures (Jellesma and Vingerhoets 2012; van Hemert et al. 2011, for reviews see Bekker et al. 2001; Vingerhoets and Bylsma 2016). These gender differences seem to emerge in middle childhood and persist during adulthood. Masculine sex-role stereotypes may discourage men to display internalising emotions via crying (Brooks 2011; Goodey 1997; Peter et al. 2001) and men display less help-seeking behaviour in response to mental illness and suffering, which has been suggested to be linked to their higher suicide rates (Johnson et al. 2012; Keohane and Richardson 2018). This reluctance to appear helpless and to attract help is possibly mirrored in the average male showing lower frequent crying behaviour than women. On the other hand, women tend to bond with others via the means of crying in stressful situations (Lane 2006). Thus, it has been suggested that due to these diverging social norms, interpersonal benefits of crying are limited to females (Cretser et al. 1982; Warner and Shields 2007). Cretser et al. (1982) demonstrated that, when asking participants about their hypothetical reactions to crying men or women, men employed a double standard: Whereas women rated crying men and women equally, men evaluated male crying generally more negatively than female crying.

\section{Study aims}

Building on the work of Vingerhoets et al. (2016) the current study aims to replicate the effect of visible tears on observers' willingness to help (H1: Perceiver will be more willing to help individuals displaying tears than individuals without tears). Extending the previous study, differences between the gender combinations will be examined, including males' reactions to crying males. Hereby, we expect to find a double standard as reported by Cretser et al. (1982); (H2: Men will indicate more willingness to help tearfully crying women than male counterparts, while women will indicate a similar willingness to help for both tear-displaying genders). Moreover, differential mediators are expected, as women may use crying to bond with others, while for men crying may merely be an expression of helplessness (H3a: Female observer's willingness to help will be influenced by perceived connectedness, helplessness, and friendliness; 
H3b: Male observer's willingness to help is expected to be only mediated by helplessness and friendliness).

\section{Methods}

This study was pre-registered (see https://aspredicted.org/ f8c54.pdf) and the preregistered procedures were followed, extended with a post hoc application of a more advanced mediation analysis for comparison. The empathy assessment mentioned in the preregistration was omitted for this paper as the current focus is on gender differences in interpersonal crying mechanisms and as it was not directly connected to the main experiment and analysis.

\section{Participants}

Participants were 140 students (50\% female) from the University of Groningen based on a power analysis for a repeated measures ANOVA with a medium effect size (see https://aspredicted.org/f8c54.pdf). Of these students, 125 were in the first-year psychology program receiving course credits for study participation. The remaining 15 students (all male) were recruited from other faculties, and received $7 €$ for participation. Participant age ranged from 18 to 34 $(M=20.58, S D=2.68)$. The study was conducted in English. The students, however, were natively German $(n=61$, $43.6 \%)$, Dutch $(n=45,32.1 \%)$, British $(n=10,7.1 \%)$ or from other countries $(n=24,17.1 \%)$. The majority of the sample were heterosexual $(n=125,89.3 \%)$, a small fraction bisexual ( $n=10,7.1 \%$ ), two participants homosexual (1.4\%), and three did not want to indicate their sexual orientation or felt not represented by any of the categories $(2.1 \%)$.

\section{Materials and apparatus}

\section{Stimulus material}

A subset of the original visual stimuli of the study by Vingerhoets et al. (2016) was used. For the present study, 32 pictures ( 2 versions of 16 photos) were selected to ensure that equal numbers of men and women were displayed. Half of the stimuli were original photos of a person crying with visible tears, the other half consisted of the same pictures with tears digitally removed. Similar to the original methodology, two sets of 16 pictures (each containing 8 men and 8 women, of which half were depicted with tears, respectively) were created to control for effects of selected images and picture order. The two sets contained images of different individuals but were constructed to be comparable in age and ethnic diversity of the depicted individuals. As in the original study, participants were only exposed to one set of pictures.

\section{Measurements}

All assessments relevant to the main effect of tears and the proposed mediators closely followed procedures laid out by Vingerhoets et al. (2016).

Stimulus ratings Four constructs were assessed with nine statements rated on a scale from 1 (=do not agree with the statement) to 7 (= full confirmation of the statement): Helplessness of the depicted person (Cronbach's $\alpha=.91$; "This person needs support"; "This person needs consolation"), Friendliness of the depicted person (Cronbach's $\alpha=.88$; "This person seems kind"; "This person seems likable"), Connectedness with the depicted person (Cronbach's $\alpha=.82$; "Ifeel connected to this person", "I feel emotionally involved with this person"; "I sympathize with this person") and Willingness to help the depicted person (Cronbach's $\alpha=.93$; "I am inclined to ask this person whether I can be of any help"; "I am inclined to comfort this person"). All constructs showed very high reliability estimates in the current sample $(N=140)$.

\section{Design}

A within-subject design with regard to the experimental stimuli was employed: participants saw pictures with and without tears. Considering the gender of the participant and of the person depicted on the photos, a $2 \times 2 \times 2$ design emerged, with participant gender as a between-subjects factor and tears/no tears as well as male/female individual displayed in the picture as within-subjects factors.

\section{Procedure}

The study followed the Helsinki protocol and was approved by the ethics board of the Psychology Department of the University of Groningen. Participants were recruited via an internal student participant platform and were tested in a lab with up to three participants in the same room. Collecting data from one subject took approximately $35 \mathrm{~min}$. Informed consent was obtained before detailed instructions on the experimental task were received. The main task was presented using a Qualtrics survey on a 19-in monitor. Three training trials were run for all participants, which employed the exact same procedure as the main task. For each trial, participants saw one photo for $5 \mathrm{~s}$, and were asked to rate three of the nine stimulus ratings after picture off-set without time constraint. This process was repeated twice for the same picture with different statements, until all nine statements were answered. In total, each picture thus was presented three times for $5 \mathrm{~s}$.

Each statement block contained items measuring different constructs (see Table 1). While the composition of 
Table 1 Statement blocks

\begin{tabular}{lll}
\hline S1-statement block 1 & S2-statement block 2 & S3-statement block 3 \\
\hline "I feel connected to this person." & "I feel emotionally involved with this person." & "I sympathize with this person." \\
"This person seems kind." & "I am inclined to ask this person whether I can be of any & "This person seems likable." \\
"This person needs support." & "This person needs consolation." & "I am inclined to comfort this person." \\
\hline
\end{tabular}

the blocks was kept constant for all participants, the blocks were presented in three different orders to control for order effects. Within subjects, the block order was kept constant across stimuli, thus all stimuli were rated in the same order. In addition, the Inclusion of Others in the Self Scale (Aron et al. 1992) that added to the connectedness assessment was shown directly following the last of the three blocks.

The main task consisted of 16 trials and participants were randomly assigned to one of six conditions: picture set $\mathrm{A}$ or B and statement block order one, two, or three. Finally, before participants were debriefed, we asked them to fill out an empathy questionnaire, which was used for a separate analysis.

\section{Data analysis}

For the analysis, IBM SPSS Statistics Version 23 was used. Statistical significance was defined as $p<.05$ two-sided, according to convention.

\section{Calculating mean ratings}

For each of the four constructs, the mean stimulus rating per picture was computed per participant (e.g., picture 1 helplessness was calculated by calculating the mean of the two helplessness statements). Then mean ratings per participant per condition (tears/no tears and male/female depicted gender) were calculated across pictures. Subsequently, descriptive statistics were obtained.

\section{Replication main effect}

Paired $t$-tests comparing the conditions of tears versus no tears were performed with Willingness to Help as the dependent variable to replicate the effect reported by Vingerhoets et al. (2016; H1). The difference in mean between the tear and no tear image conditions for all four constructs was expressed as Cohen's $d$.

\section{$2 \times 2$ design}

In order to investigate the differences between the gender combinations in the main effect of tears on Willingness to Help (H2), a repeated measures ANOVA was estimated with a within subject factor Male/Female displayed in picture, participant's gender as between-subject factor and mean willingness to help rating as the dependent variable, only including the images of crying individuals. Hereby, a significant two-way interaction indicates differences between the Image/Participant combinations. In order to obtain the needed information for hypothesis two, a plot of this interaction including $95 \%$ confidence intervals was created. For all effects, generalized partial eta squared estimates were calculated according to formulas presented by Olejnik and Algina (2003).

\section{Mediator analysis}

A more recent path analytic approach to within-subject mediation analysis by Montoya and Hayes (2017) was applied using the MEMORE macro for $\mathrm{SPSS}^{2}$. In this approach, indirect effects are estimated in one step instead of two separate steps. The mediation model by Vingerhoets et al. (2016) was calculated separately for female (H3a) and male (H3b) participants. Hereby, perceived helplessness, friendliness, and agreeableness were tested as potential mediators of the tears-elicit-help effect.

\section{Manipulation check}

To explore the differences between the two created picture sets, the three statement block orders, and participants from the two recruitment strategies, effect sizes were examined. For differences in the dependent variables between the two recruitment strategies or the two picture sets Cohen's $d$ was calculated. For differences between the statement block orders partial eta squared $\left(\eta_{P}^{2}\right)$ was determined. Whereas the recruitment strategy did not cause medium or large differences in ratings, limitations regarding the statement orders and picture sets were identified. The statement orders influenced the Connectedness rating for images displaying females with tears $\left(\eta_{P}^{2}=0.05\right)$. The differences in picture sets influenced Willingness to Help

\footnotetext{
${ }^{2}$ Results of an exact replication of the mediation analysis by Vingerhoets et al. (2016) for the full sample as well as female and male participants separately can be found in the supplement.
} 
Table 2 Descriptive statistics main constructs

\begin{tabular}{|c|c|c|c|c|c|c|c|c|}
\hline & \multicolumn{4}{|c|}{ Male image } & \multicolumn{4}{|c|}{ Female image } \\
\hline & \multicolumn{2}{|l|}{ Tears } & \multicolumn{2}{|c|}{ No tears } & \multicolumn{2}{|c|}{ Tears } & \multicolumn{2}{|c|}{ No tears } \\
\hline & $M$ & $S D$ & $M$ & $S D$ & $M$ & $S D$ & $M$ & $S D$ \\
\hline \multicolumn{9}{|l|}{ Helplessness } \\
\hline Whole sample & 4.85 & 0.84 & 3.82 & 0.88 & 5.16 & 0.73 & 4.15 & 0.85 \\
\hline Women & 5.09 & 0.82 & 3.86 & 0.88 & 5.21 & 0.81 & 4.23 & 0.91 \\
\hline Men & 4.62 & 0.80 & 3.77 & 0.87 & 5.11 & 0.65 & 4.07 & 0.79 \\
\hline \multicolumn{9}{|l|}{ Friendliness } \\
\hline Whole sample & 4.97 & 0.71 & 4.65 & 0.82 & 5.15 & 0.65 & 4.65 & 0.82 \\
\hline Women & 5.15 & 0.67 & 4.66 & 0.79 & 5.35 & 0.62 & 4.78 & 0.87 \\
\hline Men & 4.78 & 0.70 & 4.39 & 0.77 & 4.95 & 0.62 & 4.52 & 0.75 \\
\hline \multicolumn{9}{|l|}{ Connectedness } \\
\hline Whole sample & 3.97 & 0.91 & 3.52 & 0.87 & 4.18 & 0.89 & 3.52 & 0.87 \\
\hline Women & 3.94 & 0.87 & 3.16 & 0.81 & 4.21 & 0.89 & 3.53 & 0.92 \\
\hline Men & 4.00 & 0.96 & 3.44 & 0.89 & 4.16 & 0.91 & 3.51 & 0.82 \\
\hline \multicolumn{9}{|c|}{ Willingness to help } \\
\hline Whole sample & 4.47 & 0.96 & 3.56 & 1.00 & 4.96 & 0.88 & 3.93 & 0.96 \\
\hline Women & 4.72 & 0.85 & 3.63 & 0.98 & 5.05 & 0.80 & 4.05 & 0.98 \\
\hline Men & 4.20 & 1.00 & 3.48 & 1.01 & 4.86 & 0.95 & 3.81 & 0.94 \\
\hline
\end{tabular}

The total sample comprised 140 participants including 70 women and 70 men $(d=0.39)$ ratings for images of males with tears, Friendliness ratings of females displayed with tears $(d=0.35)$ and without tears $(d=0.51)$, as well as Helplessness ratings of male without tears $(d=0.39)$.

\section{Results}

\section{Descriptive statistics}

Descriptives for the four assessed constructs are summarised in Table 2.

\section{Replication main effect}

The main effect of tear display on perceivers' willingness to help the person on the picture was significant and substantial $\left(t_{(139)}=15.41, p<.001, d=1.30\right)$. Also, the effects of tears on the remaining three constructs were medium to large in magnitude $(d>0.60$, see Table 3$)$. After visual inspection we concluded that the assumptions for paired $t$-tests were reasonably well met, except that the scores for willingness to help crying individuals showed a skewed distribution. We therefore ran an additional non-parametric test to compare Willingness to Help for tears versus no tears, which showed identical results (Related-Samples Wilcoxon Signed Rank Test, $p<.001)$.

\section{$2 \times 2$ design}

Due to the nature of the data, multivariate test results as given by SPSS did not differ from univariate results. No assumptions seemed to be violated upon visual inspection.

The two-way interaction between displayed gender and participant gender proved significant $(F(1,138)=7.12$; $\left.p=.009 ; \eta_{G}^{2}=0.004\right)$. The plot of this interaction revealed that male participants report a significantly lower willingness to help males depicted with tears than females, providing support for hypothesis two (Fig. 1).

\section{Mediator analysis}

In the mediation analysis for male participants, only the indirect effect of Helplessness ( $a b=0.43 ; 95 \%$ CI0.28, 0.59) was

Table 3 Comparison tears/no tears

\begin{tabular}{lllllll}
\hline & \multicolumn{2}{l}{ Tears } & & \multicolumn{2}{l}{ No tears } & \multirow{2}{*}{ Effect size } \\
& $M$ & $S D$ & & $M$ & $S D$ & $d$ \\
\hline Whole sample & & & & & \\
Helplessness & 5 & 0.72 & & 3.98 & 0.84 & 1.3 \\
Friendliness & 5.06 & 0.6 & 4.59 & 0.71 & 0.72 \\
Connectedness & 4.08 & 0.82 & 3.41 & 0.91 & 0.77 \\
Willingness to help & 4.71 & 0.84 & 3.79 & 0.96 & 1.02 \\
\hline
\end{tabular}

$N=140 . d=$ Cohen's $d$ (see method section)

$M$ mean, $S D$ standard deviation 
Fig. 1 Two-way-interaction of image gender by participant gender with $95 \%$ confidence intervals

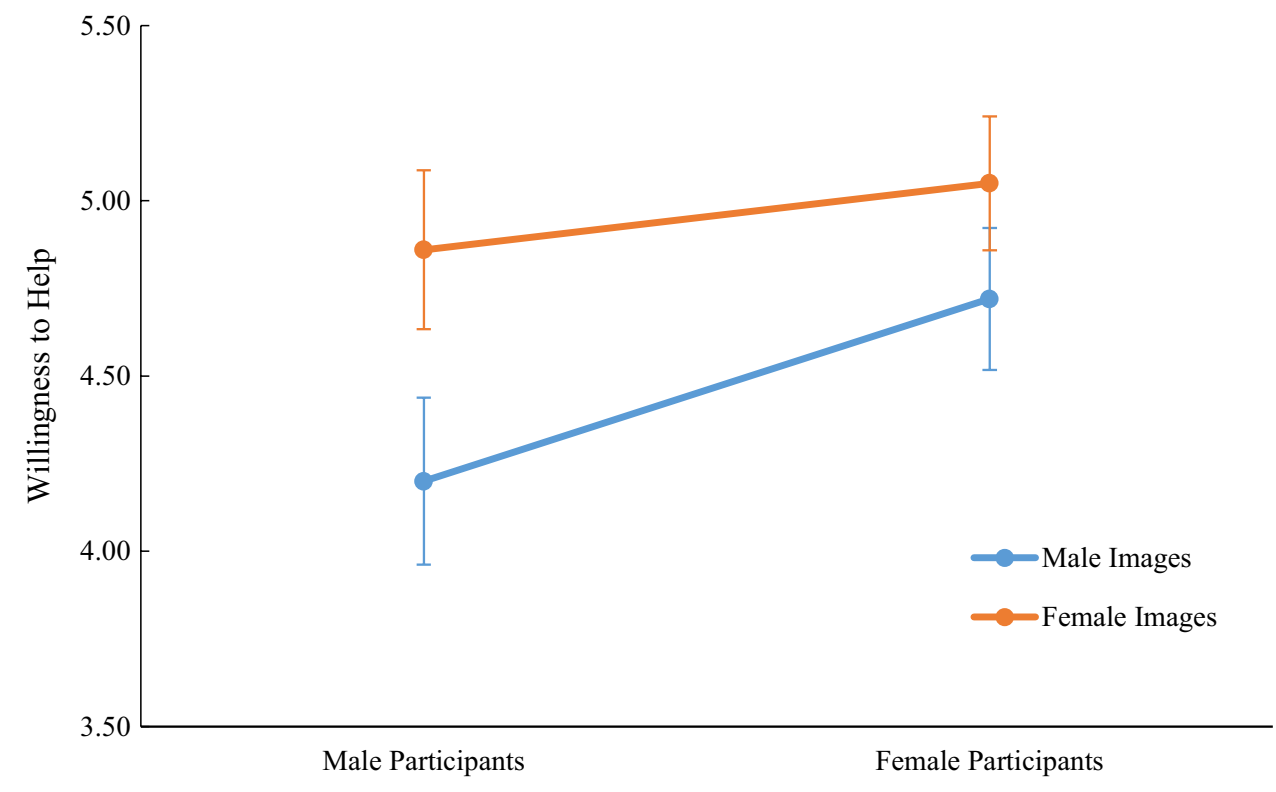

Table 4 Path-analytic mediation analysis in all gender dyads

\begin{tabular}{llll}
\hline Mediator & $\begin{array}{l}\text { Direct effect }(\mathrm{T} / \\
\mathrm{NT} \rightarrow \mathrm{WH})\end{array}$ & $\begin{array}{l}\text { Indirect effect }(\mathrm{T} / \\
\mathrm{NT} \rightarrow \mathrm{M} \rightarrow \mathrm{WH})\end{array}$ & 95\% confidence interval \\
\hline $\begin{array}{l}\text { Male participants } \\
\text { Helplessness }\end{array}$ & 0.16 & 0.43 & $0.28,0.59$ \\
Friendliness & & 0.08 & $-0.04,0.21$ \\
Connectedness & & 0.17 & $-0.03,0.40$ \\
Female participants & & & \\
Helplessness & 0.03 & 0.59 & $0.34,0.80$ \\
Friendliness & & 0.03 & $-0.09,0.13$ \\
Connectedness & & 0.34 & $0.19,0.58$ \\
\hline
\end{tabular}

${ }^{\mathrm{a}} N=70$; effects as given by SPSS macro MEMORE V2.BETA3 significant (see Table 4). For female participants, the indirect effects of both Helplessness ( $a b=0.59 ; 95 \%$ CI0.34, 0.80) and Connectedness ( $a b=0.34,95 \%$ CI $0.19 ; 0.58$ ) were significant (see Table 4). Hence, the hypothesized mediation by friendliness could not be established in either gender.

\section{Discussion}

The aim of the present study was to investigate the communicative function of adult crying. Building on previous work of Vingerhoets et al. (2016), the present study replicated the observation that tear display increases the willingness to help a crying individual using a within-subject design. New to the literature were our tests of gender differences among observers and in the displayed crying individuals which indicate that a male-male dyad is characterised by significantly lower willingness to help than all other possible gender combinations when tears are displayed. In addition, one previously proposed mediator of the relationship between display of tears and willingness to help was present for male and female participant gender, namely, perceived helplessness of the depicted individual. Additionally, perceived connectedness with the depicted individual only mediated willingness to help in female participants and perceived friendliness did not mediate this relationship in either gender.

\section{Willingness to help}

Our replication of the main effect of the display of tears on willingness to help as reported by Vingerhoets et al. (2016) increases confidence in its generalisability. The tears-elicithelp effect was detected with a similar magnitude despite some changes in the experimental set up, including (a) the composition of picture sets, (b) the item order, and (c) inclusion of male participants when displaying images of females as well as males. 
The most intriguing result lies in the two-way interaction between gender of the participant and the depicted person when considering images with visible tears: In males, the willingness to help was significantly lower for a crying male than a crying female. Women did not display this double standard. Thus, men benefited more from crying when observed by a woman than by a man. Consequently, our preregistered hypothesis that men will display more willingness to help tear-displaying women than men was supported. These results could be interpreted in line with the interpretation by Goodey (1997) that men are traditionally more discouraged from emotional display and especially crying due to cultural norms of hegemonic masculinity, i.e., cultural practices establishing males as the dominant sex.

In the same vein, it has been suggested that men learn to use help-seeking displays such as crying less frequently as it results in less received support (Addis and Mahalik 2003) and, depending on the context, comes with negative appraisals regarding the subject's competence (Fischer et al. 2013). These differing consequences might be related to gender differences in intentional up- or down-regulation of emotional displays in social situations (Rydell et al. 2003; Simons et al. 2013). However, according to the current results, men do still benefit from crying when a male observer is present, just not as much as women. A refining interpretation given by Reigeluth et al. (2016) posits that men tend to be emotionally expressive only in close relationships but not with strangers, since the latter situation might pose a threat to their reputation. Moreover, research shows that heterosexual men usually form such emotionally close ties in cross-sex friendships and romantic relationships (Coombs 1991), thus always in interaction with a female counterpart. One way to explain the current findings might be that this male supportseeking pattern is also mirrored in their willingness to give support, which would mean that men more readily turn to women for emotional support since women are more willing than men to provide it. Future studies may scrutinize expressivity, instrumentality, androgyny, and sexual orientation at the individual level, and account for the role of culture, in order to better explain the gender differences we observed.

\section{Further effects of the display of tears}

Present findings show that people are not only readier to help a crying person, but also to have a heightened perception of helplessness of him or her. This effect mostly explains the relationship between tears and increased willingness to help. Examining male and female participants separately, total mediation occurs. In female participants, the connectedness with the crying individual further mediated this relationship. Thus, helplessness might draw on the empathic capabilities of an individual, eliciting prosocial behaviour, while connectedness does so only for a female observer. Friendliness appears to not be a relevant mediator of this relationship.

Interestingly, our results support the results of the within-subject design of Vingerhoets et al. (2016), while their between-subjects design (male and female participants, females depicted) supported all three mediators. Their within-subject design (female participants, males and females depicted), however, did not support perceived friendliness as a mediator of the link between tear display and observer willingness to help, and our results were similar. Our study showed, however, that mediators might additionally depend on the gender of the depicted individual: when limiting the analysis to female images, connectedness became significant. Thus, connectedness seems to be particularly important when considering to help a female while it does not seem play a role for a male in need.

Differences in sample composition might also account for the different findings concerning the mediation effect in the present work and Vingerhoets' original within-subjects study on the one hand and Vingerhoets' between-subjects study on the other hand: While the latter one recruited participants via Amazon MTurk, both other studies, the present work and Vingerhoets et al.'s within subjects design, utilized student samples. Future studies are to determine if the resulting differences are simply due to systematic differences in the study populations, such as age or educational background. In addition, a replication of the tear-elicit-help effects in non-student, elderly, and more culturally diverse samples might provide further interesting insights.

\section{Limitations and future studies}

All results and their interpretations should be viewed with caution, considering several limitations and areas to expand on: Most importantly, the manipulation checks of the item orders and picture sets as described in the method section uncovered serious limitations. It is unknown if a similar effect might have been present in the original study and it is thus impossible to know whether the effect holds for a more balanced picture selection. A probable reason for the discrepancies produced by the two picture sets might be differences in attractiveness of the people displayed (between and within the two genders), which future studies should consider in more detail. Due to the within-subject design, we can also not rule out that some carry-over effects might have occurred, although we tried to account for them by counterbalancing the order.

Another main concern of the present study is its external validity. First, this study utilised a restricted sample: Western psychology students. Regarding a topic such as crying, which is under the influence of social norms, the role of culture can be considered as highly important 
(Becht and Vingerhoets 2002; van Hemert et al. 2011) and thus generalization to different contexts is probably limited. Moreover, the study was performed in a lab and with pictures as stimuli which constitutes a rather artificial set up. Additionally, no context for the emotion was given and therefore the urgency for help of the crying individual remained unclear. This seems particularly relevant as previous studies have indicated that the broader societal and also immediate social context strongly influences how emotional displays are judged (see Fischer et al. 2013). In a similar vein, the convincingness of the stimuli might need to be further explored. This seems especially imperative as it is conceivable that there are gender-differences in the perceived authenticity of the stimuli. Moreover, tears were only artificially removed, not added, therefore the images in displaying no tears might be seen as less authentic and produce biased results. The link between the display of tears and willingness-to-help should thus be replicated in a more naturalistic setting or while keeping the context under experimental control.

Future studies should aim at reproducing the found effect in a between-subject design and while measuring actual helping behaviour instead of the intention to help since oftentimes intention does not directly translate to behaviour (Papies 2017). Moreover, following up on the detected gender differences, studies should examine possible explanations for the difference of male-male dyads directly, and potential moderators, including cultural norms and sexual orientation. Moreover, future studies could also consider the context of crying, specifically the authenticity of tears, the perceived appropriateness of the crying reaction, and lifespan differences.

\section{Conclusion}

The current study contributes to the literature by replicating the effect of the display of tears on helping intentions, while this effect remains to be shown for actual helping behaviour. The effect, however, seems to be less potent for a male dyad than for a female or mixed dyad. Moreover, the impression of helplessness of a crying person plays a crucial role in eliciting an intention to help, mediating the main effect regardless of gender. Perceived connectedness with the crying individual, however, acts as a mediator in female observers only.

Funding This study was funded by People: Marie-Curie Actions (Grant Number FP7) to JKD.

\section{Compliance with ethical standards}

Conflict of interest All authors declare that they have no conflict of interest.

Ethical approval All procedures performed in studies involving human participants were in accordance with the ethical standards of the institutional and/or national research committee and with the 1964 Helsinki declaration and its later amendments or comparable ethical standards.

Informed consent Informed consent was obtained from all individual participants included in the study.

Open Access This article is distributed under the terms of the Creative Commons Attribution 4.0 International License (http://creativeco mmons.org/licenses/by/4.0/), which permits unrestricted use, distribution, and reproduction in any medium, provided you give appropriate credit to the original author(s) and the source, provide a link to the Creative Commons license, and indicate if changes were made.

\section{References}

Addis, M. E., \& Mahalik, J. R. (2003). Men, masculinity, and the contexts of help seeking. American Psychologist, 58(1), 5-14. https ://doi.org/10.1037/0003-066x.58.1.5.

Aron, A., Aron, E. N., \& Smollan, D. (1992). Inclusion of other in the self scale and the structure of interpersonal closeness. Journal of Personality and Social Psychology, 63(4), 596-612. https://doi. org/10.1037/0022-3514.63.4.596.

Balsters, M. J. H., Krahmer, E. J., Swerts, M. G. J., \& Vingerhoets, A. J. J. M. (2013). Emotional tears facilitate the recognition of sadness and the perceived need for social support. Evolutionary Psychology, 11(1), 148-158. https://doi.org/10.1177/1474704913 01100114

Barr, R. G., Fairbrother, N., Pauwels, J., Green, J., Chen, M., \& Brant, R. (2014). Maternal frustration, emotional and behavioural responses to prolonged infant crying. Infant Behavior and Development, 37(4), 652-664. https://doi.org/10.1016/j.infbe h.2014.08.012.

Barrett, L. F. (2018). How emotions are made: The secret life of the brain. Boston: Houghton Mifflin Harcourt.

Becht, M. C., \& Vingerhoets, A. J. J. M. (2002). Crying and mood change: A cross-cultural study. Cognition and Emotion, 16(1), 87-101. https://doi.org/10.1080/02699930143000149.

Bekker, M. H., Vingerhoets, A. J., \& Cornelius, R. R. (2001). Male and female tears: Swallowing versus shedding? In A. J. J. M. Vingerhoets \& R. R. Cornelius (Eds.), Adult crying. A biopsychosocial approach (pp. 91-114). Boca Raton: Brunner-Routledge.

Bressan, P., Colarelli, S. M., \& Cavalieri, M. B. (2009). Biologically costly altruism depends on emotional closeness among step but not half or full sibling. Evolutionary Psychology, 7(1), 118-132.

Brooks, D. J. (2011). Testing the double standard for candidate emotionality: Voter reactions to the tears and anger of male and female politicians. The Journal of Politics, 73(2), 597-615. https://doi. org/10.1017/s0022381611000053.

Bylsma, L. M., Croon, M. A., Vingerhoets, A. M., \& Rottenberg, J. (2011). When and for whom does crying improve mood? A daily diary study of 1004 crying episodes. Journal of Research in Personality, 45(4), 385-392. https://doi.org/10.1016/j. jrp.2011.04.007. 
Coombs, R. H. (1991). Marital status and personal well-being: A literature review. Family Relations: An Interdisciplinary Journal of Applied Family Studies, 40(1), 97-102. https://doi. org/10.2307/585665.

Cornelius, R. R., \& Lubliner, E. (2003). The what and why of others' responses to our tears: Adult crying as an attachment behavior. Third International Conference on the (Non) Expression of Emotions in Health and Disease, Tilburg, the Netherlands.

Cretser, G. A., Lombardo, W. K., Lombardo, B., \& Mathis, S. (1982). Reactions to men and women who cry: A study of sex differences in perceived societal attitudes versus personal attitudes. Perceptual and Motor Skills, 55(2), 479-486. https://doi.org/10.2466/ pms. 1982.55.2.479.

Crivelli, C., \& Fridlund, A. J. (2018). Facial displays are tools for social influence. Trends in Cognitive Sciences, 22(5), 388-399. https:// doi.org/10.1016/j.tics.2018.02.006.

Darwin, C. (1872). The expression of the emotions in man and animals (3rd ed.). New York: Oxford University Press.

De Waal, F. (2019). Mama's last hug: Animal emotions and what they tell us about ourselves. New York: W.W. Norton \& Company.

Denckla, C. A., Fiori, K. L., \& Vingerhoets, A. J. J. M. (2014). Development of the crying proneness scale: Associations among crying proneness, empathy, attachment, and age. Journal of Personality Assessment, 96(6), 619-631. https://doi.org/10.1080/00223 891.2014.899498.

Fischer, A. H., Eagly, A. H., \& Oosterwijk, S. (2013). The meaning of tears: Which sex seems emotional depends on the social context. European Journal of Social Psychology, 43(6), 505-515. https:// doi.org/10.1002/ejsp.1974.

Goodey, J. (1997). Boys don't cry: Masculinities, fear of crime and fearlessness. British Journal of Criminology, 37(3), 401-418. https://doi.org/10.1093/oxfordjournals.bjc.a014177.

Gračanin, A., Bylsma, L. M., \& Vingerhoets, A. M. (2018). Why only humans shed emotional tears: Evolutionary and cultural perspectives. Human Nature, 29(2), 104-133. https://doi.org/10.1007/ s12110-018-9312-8.

Hasson, O. (2009). Emotional tears as biological signals. Evolutionary Psychology, 7(3), 363-370. https://doi.org/10.1177/1474704909 00700302.

Hendriks, M. C. P., Croon, M. A., \& Vingerhoets, A. J. J. M. (2008). Social reactions to adult crying: The help-soliciting function of tears. The Journal of Social Psychology, 148(1), 22-42. https:// doi.org/10.3200/socp.148.1.22-42.

Hendriks, M., \& Vingerhoets, A. (2006). Social messages of crying faces: Their influence on anticipated person perception, emotions and behavioural responses. Cognition and Emotion, 20(6), 878-886. https://doi.org/10.1080/02699930500450218.

Jellesma, F. C., \& Vingerhoets, A. J. J. M. (2012). Crying in middle childhood: A report on gender differences. Sex Roles, 67(7-8), 412-421. https://doi.org/10.1007/s11199-012-0136-4.

Johnson, J. L., Oliffe, J. L., Kelly, M. T., Galdas, P., \& Ogrodniczuk, J. S. (2012). Men's discourses of help-seeking in the context of depression. Sociology, Health and Illness, 34(3), 345-361. https ://doi.org/10.1111/j.1467-9566.2011.01372.x.

Keohane, A., \& Richardson, N. (2018). Negotiating gender norms to support men in psychological distress. American Journal of Men's Health, 12(1), 160-171. https://doi.org/10.1177/15579 88317733093.

Lane, C. J. (2006). Evolution of gender differences in adult crying. $\mathrm{PhD}$ thesis. Arlington: University of Texas.

Masson, J. M., \& McCarthy, S. (1995). When elephants weep: The emotional lives of animals. New York: Delacorte Press.

Mizokawa, A. (2011). Young children's understanding of the interpersonal functions of apparent crying. Japanese Journal of Developmental Psychology, 22(1), 33-43.
Montoya, A. K., \& Hayes, A. F. (2017). Two-condition within-participant statistical mediation analysis: A path-analytic framework. Psychological Methods, 22(1), 6. https://doi.org/10.1037/met00 00086.

Nelson, J. K. (2005). Seeing through tears: Crying and attachment. New York: Routledge.

Olejnik, S., \& Algina, J. (2003). Generalized eta and omega squared statistics: Measures of effect size for some common research designs. Psychological Methods, 8(4), 434-447. https://doi. org/10.1037/1082-989x.8.4.434.

Papies, E. K. (2017). Situating interventions to bridge the intentionbehaviour gap: A framework for recruiting nonconscious processes for behaviour change. Social and Personality Psychology Compass, 11(7), 5. https://doi.org/10.1111/spc3.12323.

Peter, M., Vingerhoets, A. J. J. M., \& Van Heck, G. L. (2001). Personality, gender, and crying. European Journal of Personality, 15(1), 19-28. https://doi.org/10.1002/per.386.

Provine, R. R., Krosnowski, K. A., \& Brocato, N. W. (2009). Tearing: Breakthrough in human emotional signaling. Evolutionary Psychology, 7(1), 52-56. https://doi.org/10.1177/1474704909 00700107.

Reigeluth, C. S., Pollastri, A. R., Cardemil, E. V., \& Addis, M. E. (2016). 'Mad scared' versus 'I was sad': Emotional expression and response in urban adolescent males. Journal of Adolescence, 49, 232-243. https://doi.org/10.1016/j.adolescence.2016.03.004.

Rottenberg, J., Bylsma, L. M., \& Vingerhoets, A. J. J. M. (2008). Is crying beneficial? Current Directions in Psychological Science, 17, 400-404. https://doi.org/10.1111/j.1467-8721.2008.00614.

Rydell, A. M., Berlin, L., \& Bohlin, G. (2003). Emotionality, emotion regulation, and adaptation among 5-to 8-year-old children. Emotion, 3, 30-47.

Simons, G., Bruder, M., van der Löwe, I., \& Parkinson, B. (2013). Why try (not) to cry: intra- and inter-personal motives for crying regulation. Frontiers in psychology, 3, 597. https://doi.org/10.3389/ fpsyg.2012.00597.

van Hemert, D. A., van de Vijver, F. J., \& Vingerhoets, A. J. (2011). Culture and crying: Prevalences and gender differences. CrossCultural Research: The Journal of Comparative Social Science, 45(4), 399-431. https://doi.org/10.1177/1069397111404519.

Vingerhoets, A. J., \& Bylsma, L. M. (2016). The riddle of human emotional crying: A challenge for emotion researchers. Emotion Review, 8(3), 207-217. https://doi.org/10.1177/175407391558622 6.

Vingerhoets, A. J. J. M., \& Scheirs, J. (2000). Sex differences in crying: Empirical findings and possible explanations. Gender and Emotion: Social Psychological Perspectives, 2, 143-165.

Vingerhoets, A. J., Ven, N., \& Velden, Y. (2016). The social impact of emotional tears. Motivation and Emotion, 40(3), 455-463. https ://doi.org/10.1007/s11031-016-9543-0.

Warner, L. R., \& Shields, S. A. (2007). The perception of crying in women and men: Angry tears, sad tears, and the 'right way' to cry. In P. Hess (Ed.), Group dynamics and emotional expression (pp. 92-117). New York: Cambridge University Press. https://doi. org/10.1017/cbo9780511499838.006.

Wassiliwizky, E., Jacobsen, T., Heinrich, J., Schneiderbauer, M., \& Menninghaus, W. (2017). Tears falling on goosebumps: Cooccurrence of emotional lacrimation and emotional piloerection indicates a psychophysiological climax in emotional arousal. Frontiers in Psychology, 8(41), 1-15. https://doi.org/10.3389/ fpsyg.2017.00041.

Publisher's Note Springer Nature remains neutral with regard to jurisdictional claims in published maps and institutional affiliations. 